\title{
On The Ladder Bethe-Salpeter Equation
}

\author{
G. V. Efimov \\ Bogoliubov Laboratory of Theoretical Physics, \\ Joint Institute for Nuclear Research, Dubna, Russia
}

February 1, 2008

\begin{abstract}
The Bethe-Salpeter (BS) equation in the ladder approximation is studied within a scalar theory: two scalar fields (constituents) with mass $m$ interacting via an exchange of a scalar field (tieon) with mass $\mu$. The BS equation is written in the form of an integral equation in the configuration Euclidean $x$-space with the kernel which for stable bound states $M<2 m$ is a self-adjoint positive operator. The solution of the BS equation is formulated as a variational problem. The nonrelativistic limit of the $\mathrm{BS}$ equation is considered. The role of so-called abnormal states is discussed.

The analytical form of test functions for which the accuracy of calculations of bound state masses is better than $1 \%$ (the comparison with available numerical calculations is done) is determined. These test functions make it possible to calculate analytically vertex functions describing the interaction of bound states with constituents.

As a by-product a simple solution of the Wick-Cutkosky model for the case of massless bound states is demonstrated.
\end{abstract}




\section{Introduction}

The ladder Bethe-Salpeter equation (BS) is an effective if not unique instrument to study bound states within the framework of quantum field theory (see, for example, $[1,2,3,4,5])$. However, the exact analytical solution is obtained only for the Wick-Cutkosky model in a particular case when the masses of intermediate particle and bound state are equal to zero [2, 5]. For other cases analytical solutions are not found and numerical methods are applied. Numerous literature is devoted to numerical solutions of the ladder Bethe-Salpeter equation for different particles, propagators and interactions to get acceptable description of spectrum and other characteristics in nuclear and particle physics. Although computer numerical methods are very powerful, we are convinced that a simple method to get analytical solutions of the Bethe-Salpeter equation in the form of known special functions with accuracy around 0.1-1.0\% would be very useful for qualitative and semiquantative analysis of physical phenomena, the more if the accuracy can be improved. At present, the accuracy around $1 \%$ is undoubtedly acceptable in particle phenomenology at low energies.

A generally accepted approach to solve the BS equation ( I do not know exceptions ) consists in investigation of this equation in the momentum space where the BS-amplitude is expanded on a suitable basis in the Euclidean apace $\mathbf{R}^{4}$ to reduce the original four-dimensional integral equation to an infinite set of coupled one-dimensional equations which should be solved by numerical methods (see, for example, $[2,6,7]$ and many-many other papers).

The goal of this work is to improve the variational approach to the boundstate problem of two scalar particles with mass $m$ interacting through the exchange of a scalar particle with mass $\mu$ within the ladder Bethe-Salpeter equation. In addition, variational calculations are attractive because they give restrictions on computable parameters from one definite side, meanwhile approximate calculations do not indicate what side we approach to an exact value from. Variational methods were applied to the BS equation long time

ago $[8,9]$ and now they are used directly in the Hamiltonian formulation of quantum field theory (see, for example, [12]). Our idea is the following. In the configuration Euclidean $x$-space the BS equation can be rewritten as an integral equation with the kernel which for stable bound states $M<2 m$ is a self-adjoint positive symmetric operator. Thus, the problem of solution is reduced to searching eigenvalues and eigenfunctions of this kernel. As 
a result, the Bethe-Salpeter amplitude is expanded over this orthonormal system of functions and this series is nothing but the representation of the BS-amplitude over the Regge poles in the $s$-channel. The same representation of the BS-amplitude can be written in the $t$-channel. In this approach, the explicit form and normalization of vertex functions is naturally determined by the eigenfunctions. Besides, this representation makes clear the role of so-called abnormal states. Abnormal states are part of the full orthonormal system of functions. They are needed to get the correct representation of the Bethe-Salpeter amplitude over bound states. The experimental status of abnormal states is not clear up to now. I think this problem deserves special investigation.

Since the kernel is a self-adjoint positive and symmetric operator, the task to find eigenvalues and eigenfunctions can be formulated as a variational problem. It turned out that quite simple test functions describe the bound state masses with accuracy less than $1 \%$ (we compare our results with the numbers obtained in [6]).

It is interesting to discuss the connection between the Schrödinger and BS equations and to estimate qualitatively the value of the coupling constant for which relativistic corrections should be taken into account. It turned out that the nonrelativistic limit is realized for very small coupling constants

$\alpha \leq 10^{-3}$. In other words, any situations when binding energy is larger than $1 \%$ of constituent masses, require relativistic description.

\section{The Bethe-Salpeter equation in the self- adjoint form and its formal solution}

Within a simple quantum field model we shall consider the simplest case of the Yukawa interaction of charged scalar particles ("constituents") described by the field $\Phi$ with mass $m$ and neutral intermediate bosons ("tieons") described by the field $\phi$ with mass $\mu$. The Lagrangian density is

$$
L(x)=\Phi^{+}\left(\square-m^{2}\right) \Phi+\frac{1}{2} \phi\left(\square-\mu^{2}\right) \phi+g \Phi^{+} \Phi \phi .
$$

For simplicity, in the following we put $m=1$. It means that all dimensional variables ( $x$-coordinate, energy, momentum and masses) are expressed in units of the constituent mass $m$. This model is frequently used as the 
simplest pattern of QFT in many discussions, although this system is considered to be unstable from a strict point of view because the Hamiltonian is not bounded from below (see, for example, [10]). Nevertheless this model has been investigated by various methods (see, for example, [2, 1, 11, 12] and references therein).

A two-particle bound state corresponds to a solution of the homogeneous Bethe-Salpeter equation for a four-point amplitude. This equation in the ladder approximation in the configuration Euclidean $x$-space looks like

$$
\begin{aligned}
& \square\left(x_{1}, x_{2} ; x_{3}, x_{4}\right)=D\left(x_{1}-x_{2}\right) \delta\left(x_{1}-x_{3}\right) \delta\left(x_{2}-x_{4}\right) \\
& +g^{2} \iint d z_{1} d z_{2} D\left(x_{1}-x_{2}\right) S\left(x_{1}-z_{1}\right) S\left(x_{2}-z_{2}\right) \square\left(z_{1}, z_{2} ; x_{3}, x_{4}\right),
\end{aligned}
$$

where the propagators of constituents and tieons are

$$
\begin{aligned}
& S(x)=\int \frac{d p}{(2 \pi)^{4}} \cdot \frac{e^{i p x}}{1+k^{2}}=\frac{1}{(2 \pi)^{2}} \frac{1}{\sqrt{x^{2}}} \mathrm{~K}_{1}\left(\sqrt{x^{2}}\right), \\
& D(x)=\int \frac{d k}{(2 \pi)^{4}} \cdot \frac{e^{i k x}}{\mu^{2}+k^{2}}=\frac{1}{(2 \pi)^{2}} \frac{\mu}{\sqrt{x^{2}}} \mathrm{~K}_{1}\left(\mu \sqrt{x^{2}}\right) .
\end{aligned}
$$

Let us write down equation (2) in the center-of-mass frame, i.e. let us introduce the variables

$x_{1}=x+\frac{y}{2}, \quad x_{2}=x-\frac{y}{2}, \quad x_{3}=x^{\prime}+\frac{y^{\prime}}{2}, \quad x_{4}=x^{\prime}-\frac{y^{\prime}}{2}, \quad z_{1}=x^{\prime \prime}+\frac{y^{\prime \prime}}{2}, \quad z_{2}=x^{\prime \prime}-\frac{y^{\prime \prime}}{2}$.

Then

$$
\square\left(x_{1}, x_{2} ; x_{3}, x_{4}\right)=\square\left(x-x^{\prime} ; y, y^{\prime}\right)
$$

and equation (2) reads

$$
\begin{aligned}
& \square\left(x-x^{\prime} ; y, y^{\prime}\right)=\sqrt{D(y)} \delta\left(x-x^{\prime}\right) \delta\left(y-y^{\prime}\right) \sqrt{D\left(y^{\prime}\right)} \\
&+g^{2} \iint d x^{\prime \prime} d y^{\prime \prime} D(y) \Pi\left(x-x^{\prime \prime}, y-y^{\prime \prime}\right) \square\left(x^{\prime \prime}-x^{\prime} ; y^{\prime \prime}, y^{\prime}\right) \\
& \Pi\left(x-x^{\prime}, y-y^{\prime}\right)=S\left(x-x^{\prime}+\frac{y-y^{\prime}}{2}\right) S\left(x-x^{\prime}-\frac{y-y^{\prime}}{2}\right)
\end{aligned}
$$


Now let us go to the Fourier transform

$$
\square\left(x-x^{\prime} ; y, y^{\prime}\right)=\int \frac{d p}{(2 \pi)^{4}} e^{i p\left(x-x^{\prime}\right)} \square_{p}\left(y, y^{\prime}\right)
$$

and define the function

$$
\square_{p}\left(y, y^{\prime}\right)=\sqrt{D(y)} Z_{p}\left(y, y^{\prime}\right) \sqrt{D\left(y^{\prime}\right)}
$$

Then equation (4) becomes

$$
Z_{p}\left(y, y^{\prime}\right)=\delta\left(y-y^{\prime}\right)+g^{2} \int d y^{\prime \prime} K_{p}\left(y, y^{\prime \prime}\right) Z_{p}\left(y^{\prime \prime}, y^{\prime}\right)
$$

with the symmetric kernel

$$
\begin{aligned}
& K_{p}\left(y, y^{\prime}\right)=\sqrt{D(y)} \Pi_{p}\left(y-y^{\prime}\right) \sqrt{D\left(y^{\prime}\right)} \\
\Pi_{p}\left(y-y^{\prime}\right)= & \int \frac{d x}{(2 \pi)^{4}} e^{-i p x} S\left(x+\frac{y-y^{\prime}}{2}\right) S\left(x-\frac{y-y^{\prime}}{2}\right) \\
= & \int \frac{d k}{(2 \pi)^{4}} \frac{e^{i k\left(y-y^{\prime}\right)}}{\left(\left(k+\frac{p}{2}\right)^{2}+1\right)\left(\left(k-\frac{p}{2}\right)^{2}+1\right)} \\
= & \int \frac{d k}{(2 \pi)^{4}} \frac{e^{i k\left(y-y^{\prime}\right)}}{\left(k^{2}+1+\frac{p^{2}}{4}\right)^{2}-(k p)^{2}} .
\end{aligned}
$$

We want to emphasize that equation (5) represents the Bethe-Salpeter equation in the form of the integral equation with the symmetrical kernel $K_{p}\left(y, y^{\prime}\right)$. If we consider the problem of stable bound states, i.e. $p^{2}=-s=$ $-M^{2}$ and $M<2$ in (7), then the kernel $K_{p}\left(y, y^{\prime}\right)$ is the self-adjoint positive symmetrical normal operator on the functional space $\mathbf{L}^{2}$. This kernel can be represented in the form

$$
K_{p}\left(y, y^{\prime}\right)=\sum_{Q} U_{Q}(y, p) \Lambda_{Q}(s) U_{Q}(y, p), \quad\left(s=-p^{2}\right)
$$

where the functions $\Lambda_{Q}(s)$ and $U_{Q}(y, p)$ are the eigenvalues and eigenfunctions of the kernel $K_{p}\left(y, y^{\prime}\right)$, i.e.,

$$
\Lambda_{Q}(s) U_{Q}(y, p)=\int d y^{\prime} K_{p}\left(y, y^{\prime}\right) U_{Q}\left(y^{\prime}, p\right) .
$$


Below we discuss the quantum numbers $Q$.

The functions $\left\{U_{Q}(y, p)\right\}$ are orthonormal

$$
\int d y U_{Q}(y, p) U_{Q^{\prime}}(y, p)=\delta_{Q Q^{\prime}}, \quad \sum_{Q} U_{Q}(y, p) U_{Q}\left(y^{\prime}, p\right)=\delta\left(y-y^{\prime}\right)
$$

The general properties of the eigenvalues $\Lambda_{Q}$ are characterized by the traces

$$
T_{n}=\operatorname{Tr} K_{p}^{n}=\sum_{Q} \Lambda_{Q}^{n}(s)=\int \ldots \int d y_{1} \ldots d y_{n} K_{p}\left(y_{1}, y_{2}\right) \ldots K_{p}\left(y_{n}, y_{1}\right) .(10)
$$

The trace (10) for the kernel (6) is finite $T_{n}<\infty$ for $n \geq 3$ in the case $\mu=0$ and for $n \geq 2$ in the case $\mu>0$. It means that eigenvalues decrease as $\Lambda_{Q}(s) \sim \frac{1}{|Q|^{c}}$ with an appropriate constant $c$.

If the eigenfunctions and eigenvalues are found, one can write

$$
\delta\left(y-y^{\prime}\right)+g^{2} K_{p}\left(y, y^{\prime}\right)=\sum_{Q} U_{Q}(y, p)\left[1+g^{2} \Lambda_{Q}(s)\right] U_{Q}\left(y^{\prime}, p\right) .
$$

Let us represent

$$
Z_{p}\left(y, y^{\prime}\right)=\sum_{Q} U_{Q}(y, p) \tilde{Z}_{Q}(p) U_{Q}\left(y^{\prime}, p\right),
$$

then equation (9) looks like

$$
\tilde{Z}_{Q}(p)=1+g^{2} \Lambda_{Q}(s) \tilde{Z}_{Q}(p), \quad \tilde{Z}_{Q}(p)=\frac{1}{1-g^{2} \Lambda_{Q}(s)} .
$$

One gets

$$
Z_{p}\left(y, y^{\prime}\right)=\sum_{Q} U_{Q}(y, p) \frac{1}{1-g^{2} \Lambda_{Q}(s)} U_{Q}\left(y^{\prime}, p\right)
$$

and

$$
\square_{p}\left(y, y^{\prime}\right)=\sum_{Q} \sqrt{D(y)} U_{Q}(y, p) \frac{1}{1-g^{2} \Lambda_{Q}(s)} U_{Q}\left(y^{\prime}, p\right) \sqrt{D\left(y^{\prime}\right)}
$$


Finally, the solution of the Bethe-Salpeter equation (2) in the $x$-space is

$$
\begin{aligned}
& \square\left(x_{1}, x_{2} ; x_{3}, x_{4}\right)=\square\left(x-x^{\prime} ; y, y^{\prime}\right) \\
& =\sum_{Q} \int \frac{d p}{(2 \pi)^{4}} e^{-i p x} \sqrt{D(y)} U_{Q}(y, p) \frac{1}{1-g^{2} \Lambda_{Q}(s)} U_{Q}\left(y^{\prime}, p\right) \sqrt{D\left(y^{\prime}\right)} e^{i p x^{\prime}} .
\end{aligned}
$$

The standard Fourier transform of the amplitude $\square$ in the momentum space looks like

$$
\begin{aligned}
& \int \ldots \int d x_{1} d x_{2} d x_{3} d x_{4} e^{i\left(k_{1} x_{1}+k_{2} x_{2}-k_{3} x_{3}-k_{4} x_{4}\right)} \square\left(x_{1}, x_{2} ; x_{3}, x_{4}\right) \\
& =(2 \pi)^{4} \delta\left(k_{1}+k_{2}-k_{3}-k_{4}\right) \square(s, t) .
\end{aligned}
$$

Here $s=-\left(k_{1}+k_{2}\right)^{2}, \quad t=-\left(k_{1}-k_{3}\right)^{2}$, where $k_{1}, k_{2}$ and $k_{3}$ are the Euclidean momenta. Thus, the amplitude $\square$ as the solution of the Bethe-Salpeter equation (2) reads

$$
\square(s, t)=\sum_{Q} V_{Q}(q, p) \frac{1}{1-g^{2} \Lambda_{Q}(s)} V_{Q}\left(q^{\prime}, p\right)
$$

where $p=k_{1}+k_{2}, \quad q=k_{1}-k_{2}, \quad q^{\prime}=k_{3}-k_{4}$ and the vertices are

$$
V_{Q}(q, p)=i^{l} \int d y e^{-i q y} \sqrt{D(y)} U_{Q}(y, p)
$$

The mass of a bound state with quantum numbers $Q$ is defined by the equation

$$
1=g^{2} \Lambda_{Q}\left(M_{Q}^{2}\right), \quad p=\left(\mathbf{0}, i M_{Q}\right) .
$$

Formula (13) gives the Regge representation over the poles in the $s$ channel. The same representation is valid in the $t$-channel.

\section{Variational solution of the Bethe-Salpeter equation}

We start with the following observation. The usual variation approach is applied to the BS equation written in the well-known standard form (see, for example, $[8,9])$

$$
L \psi=\lambda D \psi
$$


where $L$ and $D$ are self-adjoint positive operators. The result is

$$
\lambda=\min _{\psi} \frac{(\psi L \psi)}{(\psi D \psi)} .
$$

For a test function $\Psi$ we get

$$
\lambda \leq \lambda_{0}=\frac{(\Psi L \Psi)}{(\Psi D \Psi)}
$$

However, if we rewrite equation (16) in the form

$$
\frac{1}{\lambda} \phi=\sqrt{D} \frac{1}{L} \sqrt{D} \cdot \phi, \quad \phi=\sqrt{D} \psi
$$

the variational problem looks like

$$
\frac{1}{\lambda}=\max _{\phi} \frac{\left(\phi \sqrt{D} \frac{1}{L} \sqrt{D} \phi\right)}{(\phi \phi)}=\max _{\psi} \frac{\left(\psi D \frac{1}{L} D \psi\right)}{(\psi D \psi)}
$$

and for the same test function $\Psi$ as in (18) we get

$$
\frac{1}{\lambda} \geq \frac{1}{\lambda_{1}}=\frac{\left(\Psi D \frac{1}{L} D \Psi\right)}{(\Psi D \Psi)}, \quad \lambda \leq \lambda_{1}
$$

One of the corollaries of the Cauchy inequality for a positive operator $L$ is

$$
(X Y)^{2} \leq(X L X)\left(Y \frac{1}{L} Y\right), \quad \forall X, Y \in \mathbf{L}^{2} .
$$

This inequality for $X=\Psi$ and $Y=D \Psi$ gives

$$
\frac{(\Psi D \Psi)}{\left(\Psi D \frac{1}{L} D \Psi\right)} \leq \frac{(\Psi L \Psi)}{(\Psi D \Psi)} \quad \text { and } \quad \lambda_{1} \leq \lambda_{0}
$$

Thus, the variational method is more preferable to apply to equation (19) and use (20).

Let us come back to equation (9). The kernel $K_{p}\left(y, y^{\prime}\right)$ in (6) for stable states $p^{2}=-s=-M^{2}, \quad M<2 m$ is the self-adjoint positive operator. The spectrum of this operator is bounded from above. The main preference 
of the representation (6) is that variational methods can be used to find the spectrum and eigenfunctions of this kernel. Therefore, the solution of equation (9) can be reduced to the variational problem in the form (20)

$$
\Lambda_{Q}(s)=\max _{U_{Q}} \frac{\left(U_{Q} K_{p} U_{Q}\right)}{\left(U_{Q} U_{Q}\right)}=\max _{U_{Q}} \frac{\left(U_{Q} \sqrt{D} \Pi_{p} \sqrt{D} U_{Q}\right)}{\left(U_{Q} U_{Q}\right)}
$$

It is convenient to introduce the function

$$
U_{Q}(y, p)=\sqrt{D(y)} \Psi_{Q}(y, p)
$$

Then formula (23) reads

$$
\Lambda_{Q}(s)=\max _{\Psi_{Q}} \frac{\left(\Psi_{Q} D \Pi_{p} D \Psi_{Q}\right)}{\left(\Psi_{Q} D \Psi_{Q}\right)}
$$

where

$$
\begin{aligned}
& \left(\Psi_{Q} D \Pi_{p} D \Psi_{Q}\right)=\iint d y d y^{\prime} \Psi_{Q}(y, p) D(y) \Pi_{p}\left(y-y^{\prime}\right) D\left(y^{\prime}\right) \Psi_{Q}\left(y^{\prime}, p\right), \\
& \left(\Psi_{Q} D \Psi_{Q}\right)=\int d y \Psi_{Q}(y, p) D(y) \Psi_{Q}(y, p) .
\end{aligned}
$$

The vertex (14) is defined by

$$
V_{Q}(q, p)=i^{l} \int d y e^{-i q y} D(y) \Psi_{Q}(y, p) .
$$

The representation (25) will be exploited in this paper.

Another possibility is to introduce the function

$$
U_{Q}(y, p)=\frac{\Phi_{Q}(y, p)}{\sqrt{D(y)}} .
$$

Then formula (23) becomes

$$
\Lambda_{Q}(s)=\max _{\Phi_{Q}} \frac{\left(\Phi_{Q} \Pi_{p} \Phi_{Q}\right)}{\left(\Phi_{Q} \frac{1}{D} \Phi_{Q}\right)}
$$

This representation is particularly convenient in the case of the Wick-Cutkosky model $(\mu=0)$ for which

$$
D(y)=\frac{1}{(2 \pi)^{2}} \cdot \frac{1}{y^{2}} \quad \text { and } \quad \frac{1}{D(y)}=(2 \pi)^{2} \cdot y^{2} .
$$


The variational problem in the form (25) and (28) is the key object of our investigation.

The mass of a bound state with quantum numbers $Q$ is defined by equation (15). The eigenvalue $\Lambda_{Q}(M)$ is a monotonically increasing function when the mass $M$ increases on the interval $M^{2} \in\left[0,4 m^{2}\right]$, i.e.

$$
\Lambda_{Q}\left(M_{1}^{2}\right)<\Lambda_{Q}\left(M_{2}^{2}\right) \quad \text { if } \quad M_{1}<M_{2}
$$

For a test function $\Psi$ in $(25)$ we get

$$
\Lambda_{Q}\left(M^{2}\right) \geq \Lambda_{Q}^{(v a r)}\left(M^{2}\right)
$$

It gives

$$
\frac{1}{g^{2}}=\Lambda_{Q}\left(M_{Q}^{2}\right)=\Lambda_{Q}^{(v a r)}\left(\left(M_{Q}^{(v a r)}\right)^{2}\right) \geq \Lambda_{Q}^{(v a r)}\left(M_{Q}^{2}\right) .
$$

Thus, the variational calculations in $(23,25,28)$ give the upper estimation for bound state masses

$$
M_{Q} \leq M_{Q}^{(v a r)}
$$

If we want to find the coupling constant for a given mass $M$, then

$$
\frac{1}{g_{(\text {var })}^{2}}=\Lambda_{Q}^{(v a r)}\left(M_{Q}^{2}\right) \leq \Lambda_{Q}\left(M_{Q}^{2}\right)=\frac{1}{g^{2}}
$$

and

$$
g^{2} \leq g_{(\text {var })}^{2}
$$

\subsection{Classification of states}

Let us consider the classification of states dictated by equation (9). The kernel $K_{p}\left(y, y^{\prime}\right)$ depends on the direction of the vector $p$. It is convenient to work in the frame $p=\left(\mathbf{0}, p_{4}\right)=(\mathbf{0}, i M)$.

Let us start with the case $M=0$, i.e. $p=0$. Then the ladder BetheSalpeter equation has $O(4)$ symmetry in the Euclidean configuration space and the eigenfunctions look like

$$
\begin{aligned}
& \Psi_{n \kappa\{\mu\}}(y)=T_{\mu_{1} \ldots \mu_{\kappa}}^{(\kappa)}(y) R_{n \kappa}\left(y^{2}\right), \\
& T_{\{\mu\}}^{(\kappa)}(y)=O\left(|y|^{\kappa}\right), \quad R_{n \kappa}\left(y^{2}\right)=O(1) \quad \text { for } \quad y \rightarrow 0,
\end{aligned}
$$


where $n$ and $\kappa$ are radial and orbital quantum numbers and $T_{\mu_{1} \ldots \mu_{\kappa}}^{(\kappa)}(y)$ are spherical harmonics in the space $\mathbf{R}^{4}$ (see Appendix). All states are degenerated over four-dimensional orbital quantum number $\kappa$ and the number of these states is $(\kappa+1)^{2}$ for fixed $n$ and $\kappa$.

Now let $M>0$. Then the Bethe-Salpeter equation possesses $O(3)$ symmetry and all states are degenerated over three-dimensional orbital quantum numbers $l$ which define the spin of corresponding bound states. The degeneration of the state (33) is taken off and the total number of these states can be represented

$$
(\kappa+1)^{2}=\sum_{l=0}^{\kappa}(2 l+1),
$$

i.e. after removal of degeneration, the state (33) turns into superposition of states with three-dimensional orbital momenta $l \leq \kappa$. Usually the state with $l=\kappa$ is considered as the desired bound state with spin $l$, but the states with $l<\kappa$, describing the spin $l$, are called "abnormal" ones and are not considered. From the point of wiew of the representation (33) the number $\kappa$ characterizes the behaviour of the wave function in the vicinity of zero, i.e. $\Psi_{n \kappa l\{\mu\}} \sim|y|^{\kappa}$ for $y \rightarrow 0$. Unconditionally the parameter $\kappa$ cannot be a normal quantum number, but for numerical calculations with a fixed accuracy $\kappa$ is connected with the smallness parameter because in real calculations the additional factor $|y|^{\kappa}$ leads to decreasing of integrals. These argumentations are consistent with the standard approach (see, for example, [6]), when the BS amplitude is developed over the four-dimensional hyperspherical harmonics $Z_{\kappa l m}(\chi, \theta, \phi)$ and the obtained system of equations is truncated over a "nonphysical" quantum number $\kappa$.

Thus, we can characterize a bound state by the quantum numbers $Q=$ $(n, \kappa, l, m)$ where $(l, m)$ are three-dimensional orbital quantum numbers, $n$ is a radial quantum number and $\kappa$ is connected with the behaviour of a wave function for $|y| \rightarrow 0$. The eigenvalues are degenerated over the magnetic quantum number $m$, i.e.

$$
\Lambda_{Q}(s)=\Lambda_{n \kappa l}(s) .
$$

On the other hand, one can say that the quantum number $\kappa$ is connected with "time" or the fourth coordinate component $y_{4}$. It has no special name and usually these states are called "abnormal". These states disappear in the nonrelativistic limit and usually are not considered. However, the representation of the Bethe-Salpeter amplitude (13) is not valid without these 
states and their role should be clarified.

We choose the test functions $\Psi_{Q}$ in the form

$$
\begin{aligned}
\Psi_{Q}(y, p) & =\Psi_{n \kappa l m}(y, p)=Y_{l m}(\mathbf{y}) W_{n \kappa l}\left(|\mathbf{y}|, y_{4}\right) \\
& =Y_{l m}(\mathbf{y}) R_{n \kappa l}\left(|\mathbf{y}|, y_{4}\right) F_{n \kappa l}\left(|\mathbf{y}|, y_{4}\right) .
\end{aligned}
$$

Here $Y_{l m}(\mathbf{y})=Y_{l m}\left(\mathbf{n}_{y}\right)|\mathbf{y}|^{l}$ with $\mathbf{n}_{y}=\mathbf{y} /|\mathbf{y}| . \quad Y_{l m}\left(\mathbf{n}_{y}\right)=Y_{l m}(\theta, \phi)$ is the standard three-dimensional spherical function; $R_{n \kappa l}(u, v)$ is a polynomial of the degree $(n+\kappa)$ and $F_{n \kappa l}(u, v)$ is a test function. The constant $N_{n \kappa l}$ is defined by the normalization condition

$$
\left(\Psi_{Q} D \Psi_{Q^{\prime}}\right)=\int d y D(y) \Psi_{n \kappa l m}(y, p) \Psi_{n^{\prime} \kappa^{\prime} l^{\prime} m^{\prime}}(y, p)=\delta_{l l^{\prime}} \delta_{m m^{\prime}} \delta_{n n^{\prime}} \delta_{\kappa \kappa^{\prime}}(35)
$$

The vertex (14) takes the form

$$
\begin{aligned}
V_{Q}(q, p) & =V_{n \kappa l m}(q, p)=i^{l} \int d y e^{-i q y} D(y) Y_{l m}(\mathbf{y}) W_{n \kappa l}\left(|\mathbf{y}|, y_{4}\right) \\
& =Y_{l m}(\mathbf{q}) V_{n \kappa l}(q, p) .
\end{aligned}
$$

where

$$
V_{n \kappa l}(q, p)=\iint d \mathbf{y} d y_{4}|\mathbf{q}|^{l}|\mathbf{y}|^{l} P_{l}\left(\mathbf{n}_{q} \mathbf{n}_{y}\right) e^{-i\left(\mathbf{q} \mathbf{y}+q_{4} y_{4}\right)} D\left(\sqrt{\mathbf{y}^{2}+y_{4}^{2}}\right) W_{n \kappa l}\left(|\mathbf{y}|, y_{4}\right)
$$

Finally, the BS amplitude (13) looks like

$$
\square(s, t)=\sum_{n \kappa l} \frac{2 l+1}{4 \pi} V_{n \kappa l}(q, p) \frac{1}{1-g^{2} \Lambda_{n \kappa l}(s)} V_{n \kappa l}\left(q^{\prime}, p\right)
$$

The test function $F(u, v)$ contains variational parameters. We choose the test function in the following simplest form

$$
\begin{aligned}
& W(y)=W\left(|\mathbf{y}|,\left|y_{4}\right|\right)=e^{-a \sqrt{\mathbf{y}^{2}+b y_{4}^{2}}}, \\
& D_{3}(y)=D_{3}\left(|\mathbf{y}|,\left|y_{4}\right|\right)=\int \frac{d k}{(2 \pi)^{4}} \cdot \frac{e^{i k y}}{\left(a^{2}+\mathbf{k}^{2}+b k_{4}^{2}\right)^{3}},
\end{aligned}
$$

where $a$ and $b$ are variational parameters.

Polynomials $R_{n \kappa l}(u, v)$ have to provide the orthogonality condition (35). 


\section{Nonrelativistic limit}

\subsection{The "Bethe-Salpeter form" of the nonrelativistic Schrödinger equation}

First of all let us represent the nonrelativistic Schrödinger equation in the "Bethe-Salpeter form". Bound states of two particles with masses $m=1$ interacting via an attractive potential are described by the equation

$$
H \psi(\mathbf{x})=\left(\mathbf{p}^{2}-\alpha V(r)\right) \psi(\mathbf{x})=-\epsilon \psi(\mathbf{x}) .
$$

where $\alpha$ is the coupling constant, $\epsilon$ is the binding energy and

$$
\mathbf{x} \in \mathbf{R}^{3}, \quad r=\sqrt{\mathbf{x}^{2}}, \quad \mathbf{p}^{2}=-\partial_{\mathbf{x}}{ }^{2} .
$$

The function $V(r)>0$ is supposed to be positive. For the Yukawa potential $V(r)=\frac{e^{-\mu r}}{r}$.

Let us rewrite equation (39) in the form

$$
\left(\mathbf{p}^{2}+\epsilon\right) \psi(\mathbf{x})=\alpha V(r) \psi(\mathbf{x}), \quad \psi(\mathbf{x})=\alpha \frac{1}{\mathbf{p}^{2}+\epsilon} \cdot V(r) \cdot \psi(\mathbf{x})
$$

and let us introduce the function

$$
\phi(\mathbf{x})=\sqrt{V(r)} \psi(\mathbf{x})
$$

Then we get

$$
\phi(\mathbf{x})=\alpha \sqrt{V(r)} \cdot \frac{1}{\mathbf{p}^{2}+\epsilon} \cdot \sqrt{V(r)} \phi(\mathbf{x})=\alpha \int d \mathbf{x}^{\prime} S_{\epsilon}\left(\mathbf{x}, \mathbf{x}^{\prime}\right) \phi\left(\mathbf{x}^{\prime}\right) .
$$

Here the kernel $S_{\epsilon}\left(\mathbf{x}, \mathbf{x}^{\prime}\right)$ for $\epsilon>0$ is the self-adjoint symmetric positive operator

$$
\begin{aligned}
S_{\epsilon}\left(\mathbf{x}, \mathbf{x}^{\prime}\right) & =\sqrt{V(r)} \cdot \frac{1}{-\partial_{\mathbf{x}}{ }^{2}+\epsilon} \delta\left(\mathbf{x}-\mathbf{x}^{\prime}\right) \cdot \sqrt{V\left(r^{\prime}\right)} \\
& =\sqrt{V(r)} \cdot \Pi_{\epsilon}\left(\mathbf{x}-\mathbf{x}^{\prime}\right) \cdot \sqrt{V\left(r^{\prime}\right)}, \\
\Pi_{\epsilon}\left(\mathbf{x}-\mathbf{x}^{\prime}\right) & =\int \frac{d \mathbf{k}}{(2 \pi)^{3}} \frac{e^{-i \mathbf{k}\left(\mathbf{x}-\mathbf{x}^{\prime}\right)}}{\mathbf{k}^{2}+\epsilon}=\frac{1}{4 \pi} \cdot \frac{e^{-\sqrt{\epsilon}\left|\mathbf{x}-\mathbf{x}^{\prime}\right|}}{\left|\mathbf{x}-\mathbf{x}^{\prime}\right|} .
\end{aligned}
$$


The solution of the Schrödinger equation (39) is equivalent to the variational problem which can be written in different forms

$$
1=\alpha \Lambda(\epsilon)=\alpha \max _{\phi} \frac{\left(\phi S_{\epsilon} \phi\right)}{(\phi \phi)}=\alpha \max _{\psi} \frac{\left(\psi V \Pi_{\epsilon} V \psi\right)}{(\psi V \psi)}=\alpha \max _{\varphi} \frac{\left(\varphi \Pi_{\epsilon} \varphi\right)}{\left(\varphi \frac{1}{V} \varphi\right)}
$$

We will show that in nonrelativistic limit $M \rightarrow 2 m$ the variational problem (25) is reduced to (41).

\subsection{The nonrelativistic limit in the Bethe-Salpeter equa- tion}

Now let us come back to the Bethe-Salpeter equation in the form (28) and consider the polarization operator $\Pi_{p}\left(y-y^{\prime}\right)$

$$
\Pi_{p}\left(y-y^{\prime}\right)=\int \frac{d \mathbf{k}}{(2 \pi)^{3}} \int \frac{d k_{4}}{2 \pi} \frac{e^{-i \mathbf{k}\left(\mathbf{y}-\mathbf{y}^{\prime}\right)-i k_{4}\left(y_{4}-y_{4}^{\prime}\right)}}{\left(\mathbf{k}^{2}+k_{4}^{2}+1-\frac{M^{2}}{4}\right)^{2}+M^{2} k_{4}^{2}}
$$

where $p=(\mathbf{0}, i M)$ and $(p k)=i M k_{4}$.

For a state $\Psi(y, p)$ we have and

$$
\left(\Psi D \Pi_{p} D \Psi\right)=\int \frac{d \mathbf{k}}{(2 \pi)^{3}} \int \frac{d k_{4}}{2 \pi} \cdot \frac{\tilde{\Psi}^{2}\left(\mathbf{k}, k_{4}\right)}{\left(\mathbf{k}^{2}+k_{4}^{2}+\Delta\right)^{2}+M^{2} k_{4}^{2}} .
$$

Here $\Delta=1-\frac{M^{2}}{4}$ and

$$
\tilde{\Psi}\left(\mathbf{k}, k_{4}\right)=\int d y e^{i p y} D(y) \Psi\left(\mathbf{y}, y_{4}\right) .
$$

In the nonrelativistic limit

$$
M \rightarrow 2, \quad \Delta=1-\frac{M^{2}}{4} \rightarrow 0 .
$$

Introducing new variables $\mathbf{k}=\sqrt{\Delta} \mathbf{q}$ and $k_{4}=\Delta q_{4}$ one can get in the limit (42)

$$
\left(\Psi D \Pi_{P} D \Psi\right)=\sqrt{\Delta} \int \frac{d \mathbf{q}}{(2 \pi)^{3}} \int \frac{d q_{4}}{2 \pi} \cdot \frac{\tilde{\Psi}^{2}\left(\sqrt{\Delta} \mathbf{q}, \Delta q_{4}\right)}{\left(\mathbf{q}^{2}+\Delta q_{4}^{2}+1\right)^{2}+4 q_{4}^{2}}
$$




$$
\begin{aligned}
& \rightarrow \sqrt{\Delta} \int \frac{d \mathbf{q}}{(2 \pi)^{3}} \int \frac{d q_{4}}{2 \pi} \frac{\tilde{\Psi}^{2}(\sqrt{\Delta} \mathbf{q}, 0)}{\left(\mathbf{q}^{2}+1\right)^{2}+4 q_{4}^{2}} \\
& =\frac{1}{4} \sqrt{\Delta} \int \frac{d \mathbf{q}}{(2 \pi)^{3}} \frac{\tilde{\Psi}^{2}(\sqrt{\Delta} \mathbf{q}, 0)}{\mathbf{q}^{2}+1}=\frac{1}{4} \int \frac{d \mathbf{k}}{(2 \pi)^{3}} \frac{\Psi^{2}(\mathbf{k}, 0)}{\mathbf{k}^{2}+\Delta}
\end{aligned}
$$

The test function is chosen in the form $\Psi(y, p)=\Psi(\mathbf{y}, 0)=\psi(\mathbf{y})$. It leads to

$$
\begin{aligned}
\tilde{R}(\mathbf{p}, 0) & =\int d \mathbf{y} \int d y_{4} e^{-i \mathbf{p y}} D(y) \psi(\mathbf{y})=\frac{1}{4 \pi} \int d \mathbf{y} \frac{e^{-\mu|\mathbf{y}|}}{|\mathbf{y}|} e^{-i \mathbf{p y}} \psi(\mathbf{y}) \\
& =\frac{1}{4 \pi} \int d \mathbf{y} e^{-i \mathbf{p y}} V(|\mathbf{y}|) \psi(\mathbf{y})
\end{aligned}
$$

and

$$
(\Psi D \Psi)=\iint d \mathbf{y} d y_{4} D(y) \psi^{2}(\mathbf{y})=\frac{1}{4 \pi} \int d \mathbf{y} \frac{e^{-\mu|\mathbf{y}|}}{|\mathbf{y}|} \psi^{2}(\mathbf{y})=\frac{1}{4 \pi}(\psi V \psi)
$$

The binding energy equals

$$
\Delta=(2-M) \cdot\left(1-\frac{2-M}{4}\right)=\epsilon\left[1-\frac{\epsilon}{4}\right] \approx \epsilon
$$

Collecting all these formulas we obtain (41)

$$
1=\lambda(\epsilon)=\alpha \max _{\psi} \frac{\left(\psi V \Pi_{\epsilon} V \psi\right)}{(\psi V \psi)}
$$

where $\alpha$ is connected with the coupling constant $g$ in the following way:

$$
\alpha=\frac{g^{2}}{16 \pi}=\frac{g^{2}}{16 \pi m^{2}}=\frac{\lambda}{4}, \quad \lambda=\frac{g^{2}}{4 \pi m^{2}} .
$$

The main result for the solution of the variational problem of the relativistic Bethe-Salpeter equation (25) for small binding energy $\epsilon \ll 1$ is that it is convenient to choose the test function $\Psi$ in the form

$$
\tilde{\Psi}(p)=\tilde{\Psi}\left(\sqrt{\Delta} a \mathbf{p}, \Delta b p_{4}\right)
$$

where $a$ and $b$ are variational parameters. 


\section{The Wick-Cutkosky model $\mu=0$}

\subsection{The mass $M_{Q}=0$ in the Wick-Cutkosky model}

As a by-product of the representation (8) equation (9) can be easily solved in the case $M_{Q}=0$ (see $\left.[1,5]\right)$. Equation (9) reads

$$
U=g^{2} \Lambda U=g^{2} K U
$$

or

$$
U(y)=\frac{g^{2}}{(2 \pi)^{2}} \frac{1}{\sqrt{y^{2}}} \int d y^{\prime} \Pi_{0}\left(y-y^{\prime}\right) \frac{1}{\sqrt{\left(y^{\prime}\right)^{2}}} U\left(y^{\prime}\right)
$$

We look for the solution in the form $U(y)=\sqrt{y^{2}} \Phi\left(y^{\prime}\right)$. Equation (45) reads

$$
y^{2} \Phi(y)=\frac{g^{2}}{(2 \pi)^{2}} \int d y^{\prime} \Pi_{0}\left(y-y^{\prime}\right) \Phi\left(y^{\prime}\right)
$$

and in the momentum space

$$
-\square_{k} \tilde{\Phi}(k)=\frac{4 \alpha}{\pi} \cdot \frac{1}{\left(k^{2}+1\right)^{2}} \tilde{\Phi}(k), \quad \alpha=\frac{g^{2}}{16 \pi} .
$$

The states are degenerated over quantum numbers $m$ and $\kappa=l$. Let us extract the angular variables

$$
\tilde{\Phi}(k)=T_{\mu_{1} \ldots \mu_{l}}^{(l)}(k) A\left(k^{2}\right)
$$

where $T_{\mu_{1} \ldots \mu_{l}}^{(l)}(k)$ is the spherical function in the four-dimension space (see Appendix) and satisfies the correlations (60)

$$
\square_{k} T_{\mu_{1} \ldots \mu_{l}}^{(l)}(k)=0, \quad\left(k \frac{\partial}{\partial k}\right) T_{\mu_{1} \ldots \mu_{l}}^{(l)}(k)=l T_{\mu_{1} \ldots \mu_{l}}^{(l)}(k) .
$$

Then equation (46) reads with $k^{2}=u \in[0, \infty]$

$$
u A^{\prime \prime}(u)+(2+l) A^{\prime}(u)+\frac{\alpha}{\pi} \frac{1}{(u+1)^{2}} A(u)=0 .
$$


Let us introduce new variables

$$
t=\frac{u-1}{u+1} \in[-1,1], \quad A(u)=(1-t)^{l+1} B(t) .
$$

We get

$$
\left(1-t^{2}\right) B^{\prime \prime}(t)-2(2+l) t B^{\prime}(t)+\left(\frac{\alpha}{\pi}-l^{2}-3 l-2\right) B(t)=0
$$

The solution is the Jacobi polynomial $B(t)=P_{n}^{(l+1, l+1)}(t)$ with

$$
\frac{\alpha}{\pi}-l^{2}-3 l-2=n(n+2 l+3)
$$

and finally

$$
\alpha_{n l}=\pi(n+l+1)(n+l+2)
$$

\subsection{Variation calculations}

For the Wick-Cutcosky model we perform variational calculations for the lowest and first excited states and compare our numerical results with the numbers obtained in [6].

\subsubsection{The lowest state}

For the lowest state the test function is chosen in the form

$$
\Psi_{0}(y, p)=W\left(|\mathbf{y}|,\left|y_{4}\right|\right)=e^{-a \sqrt{\mathbf{y}^{2}+b y_{4}^{3}}},
$$

where $a$ and $b$ are variational parameters. Then

$$
\begin{aligned}
V_{0}(k, p) & =\int d y D(y) W\left(|\mathbf{y}|,\left|y_{4}\right|\right) e^{-i k y}=\int \frac{d y}{(2 \pi)^{2} y^{2}} e^{-a \sqrt{\mathbf{y}^{2}+b y_{4}^{2}}-i \mathbf{k y}-i k_{4} y_{4}} \\
& =\frac{a b+\sqrt{a^{2} b+u b+v}}{\left[u+v+a^{2}(1+b)+2 a \sqrt{a^{2} b+u b+v}\right] \sqrt{a^{2} b+u b+v}} \\
& =R(u, v ; a, b), \quad u=\mathbf{k}^{2}, \quad v=k_{4}^{2}
\end{aligned}
$$

and

$$
(\Psi D \Psi)=\int \frac{d y}{(2 \pi)^{2} y^{2}} e^{-2 a \sqrt{\mathbf{y}^{2}+b y_{4}^{2}}}=\frac{1}{4 a^{2}(1+\sqrt{b})}
$$


The eigenvalue $\Lambda(M)$ is defined by

$$
g^{2} \Lambda\left(M^{2}\right)=\alpha \max _{a, b} \frac{8}{\pi^{2}} \int_{0}^{\infty} d k k^{2} \int_{0}^{\infty} d v \cdot \frac{4 a^{2}(1+\sqrt{b}) R^{2}\left(k^{2}, v^{2} ; a, b\right)}{\left(k^{2}+v^{2}+\Delta\right)^{2}+4(1-\Delta) v^{2}}(51)
$$

The value of $M$ is defined by equation (15). It is instructive to compare the Bethe-Salpeter mass (51) with the bound state mass of the nonrelativistic Schrödinger equation for the Coulomb potential

$$
\begin{aligned}
& \epsilon=2-M=\frac{\alpha^{2}}{4}, \quad \frac{1}{\alpha}=\frac{1}{\sqrt{8\left(1-\frac{M}{2}\right)}}=\frac{1}{\alpha_{S c h}}, \\
& \epsilon_{e m}=\frac{\alpha_{e m}^{2}}{4}=1.33 \ldots \cdot 10^{-5}, \quad \alpha_{e m}=\frac{1}{137}
\end{aligned}
$$

The numerical results are represented in Table 1 where we have used $a=$ $\sqrt{\Delta} a_{1}$ and $b=\Delta b_{1}$.

Table 1. The lowest state in the Wick-Cutkosky model. The coupling constant $\lambda=4 \alpha$ for different masses $M / m$ and comparison $1 / \alpha$ with the nonrelativistic value $1 / \alpha_{S c h}$.

\begin{tabular}{|c||c|c||c||c|c||c|c|}
\hline$\frac{M}{m}$ & $\frac{\epsilon}{2 m}$ & $\frac{1}{\alpha_{S c h}}$ & $\frac{1}{\alpha}$ & $a_{1}$ & $b_{1}$ & $\lambda=4 \alpha$ & $\lambda_{T j}[6]$ \\
\hline & & & & & & \\
0.000 & 1.00 & 0.3535 & 0.1586 & 0.76 & 1.0 & 25.21 & 25.13 \\
0.500 & 0.75 & 0.4082 & 0.1671 & 0.78 & 1.0 & 23.93 & \\
1.000 & 0.50 & 0.5000 & 0.1994 & 0.80 & 1.0 & 20.06 & 20.01 \\
1.500 & 0.25 & 0.7071 & 0.3023 & 0.84 & 1.3 & 13.23 & \\
1.900 & 0.05 & 1.5811 & 0.8901 & 0.88 & 2.1 & 4.493 & 4.483 \\
1.999 & 0.0005 & 15.811 & 13.993 & 1.00 & 4.5 & 0.285 & 0.285 \\
$2-\frac{\alpha_{e m}^{2}}{4}$ & $\frac{\alpha_{e m}^{2}}{8}$ & 137 & 133.9 & 1.0 & 6.0 & 0.0299 & \\
\hline
\end{tabular}

One can see that the variational calculations practically coincide with the numerical results obtained in [6].

It is important to remark that the nonrelativistic regime, when the Schrödinger equation can be used, is realized for very small binding energies $\epsilon \leq 10^{-4}$. 


\subsubsection{The first orbital exited states}

The test functions for the "normal" state with $\kappa=0, l=1$ and for "abnormal" state with $\kappa=1, l=0$ look like

$$
\Psi^{(n)}(y, p)=\mathbf{y} W\left(|\mathbf{y}|,\left|y_{4}\right|\right), \quad \Psi^{(a)}(y, p)=y_{4} W\left(|\mathbf{y}|,\left|y_{4}\right|\right)
$$

where the test function $W$ is the first function in (38). One can get after simple calculations

$$
\begin{aligned}
\mathbf{V}^{(n)}(k, p) & =i \int d y \mathbf{y} D(y) W\left(|\mathbf{y}|,\left|y_{4}\right|\right) e^{-i k y} \\
& =-2 \mathbf{k} \frac{\partial}{\partial u} R(u, v ; a, b)=-2 \mathbf{k} R_{u}(u, v ; a, b) \\
V^{(a)}(k, p) & =\int d y y_{4} D(y) W\left(|\mathbf{y}|,\left|y_{4}\right|\right) e^{-i k y} \\
& =-2 k_{4} \frac{\partial}{\partial v} \tilde{R}(u, v ; a, b)=-2 k_{4} R_{v}(u, v ; a, b),
\end{aligned}
$$

where the function $R$ is defined by (50), and

$$
\begin{aligned}
\left(\Psi^{(n)} D \Psi^{(n)}\right) & =\int \frac{d y \mathbf{y}^{2}}{(2 \pi)^{2} y^{2}} e^{-2 a \sqrt{\mathbf{y}^{2}+b y_{4}^{2}}}=\frac{3}{16 a^{4}} \cdot \frac{2+\sqrt{b}}{(1+\sqrt{b})^{2}} \\
\left(\Psi^{(a)} D \Psi^{(a)}\right) & =\int \frac{d y y_{4}^{2}}{(2 \pi)^{2} y^{2}} e^{-2 a \sqrt{\mathbf{y}^{2}+b y_{4}^{2}}}=\frac{3}{16 a^{4}} \cdot \frac{1}{\sqrt{b}(1+\sqrt{b})^{2}}
\end{aligned}
$$

The eigenvalue $\Lambda\left(M^{2}\right)$ is defined by

$$
\begin{aligned}
& g^{2} \Lambda^{(n)}(M)=\alpha \max _{a, b} \frac{8}{\pi^{2}} \int_{0}^{\infty} d k k^{2} \int_{0}^{\infty} d v \cdot \frac{16 a^{4}(1+\sqrt{b})^{2} 4 k^{2} R_{u}^{2}\left(k^{2}, v^{2} ; a, b\right)}{3(2+\sqrt{b})\left(k^{2}+v^{2}+1-\frac{M^{2}}{4}\right)^{2}+M^{2} v^{2}}, \\
& g^{2} \Lambda^{(a)}(M)=\alpha \max _{a, b} \frac{8}{\pi^{2}} \int_{0}^{\infty} d k k^{2} \int_{0}^{\infty} d v \cdot \frac{16 a^{4} \sqrt{b}(1+\sqrt{b})^{2} 4 q^{2} R_{v}^{2}\left(k^{2}, v^{2} ; a, b\right)}{3\left(k^{2}+v^{2}+1-\frac{M^{2}}{4}\right)^{2}+M^{2} v^{2}} .
\end{aligned}
$$

The value of $M$ is defined by equation (15). The nonrelativistic binding energy of the bound state with $l=1$ is

$$
\epsilon=2-M=\frac{\alpha^{2}}{16}, \quad \text { and } \quad \frac{1}{\alpha}=\frac{1}{\sqrt{32\left(1-\frac{M}{2}\right)}}=\frac{1}{\alpha_{S c h}}, \quad \alpha_{e m}=\frac{1}{137}
$$


The numerical results are represented in Table 2 where $a=\sqrt{\Delta} a_{1}$ and $b=$ $\Delta b_{1}$. Here we should remark that the calculations in [6] contain a mistake for $l \geq 1$. For the state with $l=1$ factor $\frac{1}{2}$ should be introduced to get the correct value.

Table 2. The first excited states in the Wick-Cutkosky model. The coupling constant $\lambda=4 \alpha$ for different masses $M / m$ and comparison $1 / \alpha$ with the nonrelativistic value $1 / \alpha_{S c h}$.

\begin{tabular}{|c||c|c||c||c|c||c|r||r|r|r|}
\hline \multicolumn{1}{|l||}{} & \multicolumn{5}{c||}{ Vector particles $l=1$} & \multicolumn{2}{c||}{ "Abnormal state" } \\
\hline \multicolumn{1}{|l||}{$\frac{M}{m}$} & $\frac{\epsilon}{2 m}$ & $\frac{1}{\alpha_{S c h}}$ & $\frac{1}{\alpha}$ & $a_{1}$ & $b_{1}$ & $\lambda_{v}=4 \alpha$ & $\lambda_{T j}$ & $\lambda_{s}$ & $a_{1}$ & $b_{1}$ \\
& & & & & & & & & \\
\hline & & & & & & & & & & \\
0.000 & 1.00 & 0.176 & .0529 & 0.80 & 1.00 & 75.62 & 80 & 75.55 & 0.85 & 1.0 \\
1.500 & 0.75 & 0.204 & 0.0559 & 0.85 & 1.00 & 71.47 & & 72.78 & 0.85 & 1.0 \\
1.500 & 0.50 & 0.250 & 0.0676 & 0.81 & 1.20 & 59.19 & 63.5 & 64.27 & 0.90 & 1.1 \\
1.900 & 0.05 & 0.353 & 0.1059 & 0.87 & 1.48 & 37.77 & & 48.55 & 0.94 & 1.4 \\
1.999 & 0.0005 & 7.906 & 0.3409 & 0.89 & 2.56 & 11.73 & 16 & 25.63 & 1.05 & 2.85 \\
$2-\frac{\alpha_{e m}^{2}}{16}$ & $\frac{\alpha_{e m}^{2}}{32}$ & 137 & 133.9 & 0.95 & 5.00 & 0.616 & 5 & 8.98 & 1.30 & 14.0 \\
\hline
\end{tabular}

One can see that "abnormal states" have practically the same mass as "normal" states in the strong coupling regime when the mass defect is large. If the coupling constant is small the "abnormal" masses are much more then "normal" masses. In addition we can repeat that the nonrelativistic regime is realized for very small binding energies.

\section{Massive tieons $\mu>0$}

In this section we perform variational calculations in the case $\mu>0$ for the lowest and the first excited states and compare our numerical results with the numbers obtained in [6]. 


\subsection{The lowest state}

For the lowest state we choose the test function in the form

$$
\Psi(y, p)=D_{3}(y, a, b)=\int \frac{d k}{(2 \pi)^{4}} \cdot \frac{e^{i k y}}{\left(a^{2}+\mathbf{k}^{2}+b k_{4}^{2}\right)^{3}}
$$

Here $a$ and $b$ are variational parameters.

The direct calculations give

$$
\begin{gathered}
(\Psi D \Psi)=\int d y D(y) D_{3}^{2}(y, a, b)=\frac{1}{2(4 \pi)^{4}} \cdot Z(\mu, a, b) \\
Z(\mu, a, b)=\iint_{0}^{1} \frac{d t d u u^{3} t(1-t)}{\sqrt{b(b u+1-u)}\left(a^{2} u+\mu^{2}(1-u) t(1-t)\right)^{3}} \\
\int d y D(y) \Psi(y) e^{i k y}=\int d y D(y) D_{3}(y, a) e^{i k y}=\frac{1}{2(4 \pi)^{4}} \cdot V(r, \theta, a, b), \\
V(r, \theta, a, b)=\int_{0}^{1} \frac{d t t^{2}}{\sqrt{b t+1-t}} \cdot \frac{1}{\left[a^{2} t+\mu^{2}(1-t)+t(1-t) r^{2}\left(\cos ^{2}(\theta)+\frac{\sin ^{2}(\theta) b}{b t+1-t}\right)\right]^{2}},
\end{gathered}
$$

where we put $|\mathbf{k}|^{2}=r^{2} \cos ^{2}(\theta)$ and $k_{4}^{2}=r^{2} \sin ^{2}(\theta)$.

The mass of the lowest state is defined by the variational equation (25). Our formulation of the problem is to find the coupling constant $\lambda=4 \alpha$ for given $\mu=\frac{\mu}{m}$ and $M=\frac{M}{m}$, then we get

$$
\lambda=\min _{a, b} \frac{\pi^{2} Z(\mu, a, b)}{J(\mu, M, a, b)}
$$

where

$$
J(\mu, M, a, b)=\int_{0}^{\infty} d r \int_{0}^{\frac{\pi}{2}} d \theta \frac{r^{3} \cos ^{2}(\theta) V^{2}(r, \theta, a, b)}{\left(r^{2}+1-\frac{M^{2}}{4}\right)^{2}+M^{2} r^{2} \sin ^{2}(\theta)}
$$

The numerical results are listed in Table3.

Table 3. The lowest states in the case $\mu>0$. 


\begin{tabular}{|c|c||c|c||c|c|c||c|}
\hline$\frac{\mu}{m}$ & $\frac{M}{m}$ & $\lambda=4 \alpha$ & $\begin{array}{c}a \\
b=1\end{array}$ & $\lambda=4 \alpha$ & $a$ & $b$ & $\lambda_{T j}$ \\
& & & & & & \\
1.000 & 0.000 & 42.960 & 1.27 & 42.960 & 1.27 & 1.00 & 42.96 \\
1.000 & 1.000 & 36.945 & 1.15 & 36.940 & 1.27 & 1.10 & 36.94 \\
1.000 & 1.900 & 17.293 & 0.66 & 17.252 & 0.70 & 1.87 & 17.23 \\
1.000 & 1.999 & 10.294 & 0.41 & 10.273 & 0.44 & 2.35 & 10.25 \\
& & & & & & & \\
0.100 & 0.000 & 25.802 & 1.02 & 25.802 & 1.02 & 1.00 & 25.80 \\
0.100 & 1.000 & 20.692 & 0.89 & 20.680 & 0.91 & 1.20 & 20.68 \\
0.100 & 1.900 & 5.3843 & 3.50 & 5.262 & 0.38 & 2.22 & 5.227 \\
0.100 & 1.999 & 1.0789 & 0.089 & 1.054 & 0.089 & 9.00 & 1.043 \\
& & & & & & & \\
0.001 & 0.000 & 25.133 & 1.00 & 25.133 & 1.00 & 1.00 & 25.13 \\
0.001 & 1.000 & 20.023 & 0.87 & 20.013 & 0.87 & 1.20 & 20.01 \\
0.001 & 1.900 & 4.6904 & 0.32 & 4.501 & 0.35 & 4.20 & 4.483 \\
0.001 & 1.999 & 0.3523 & 0.031 & 0.2900 & 0.04 & 150 & 0.289 \\
& & & & & & \\
\hline
\end{tabular}

In Table 4 we listed the results of calculations for two cases: (1) one parameter $a$ with $b=1$ and (2) two parameters $a$ and $b$. One can see that only for small binding energies there is a remarkable difference in numbers. Our numbers are quite close to the numerical results obtained in [6].

\subsection{The first excited states}

For the first excited states we choose the test functions for "normal" $(n)$ and "abnormal" (a) states in the form

$$
\Psi_{\mu}(y, p)=y_{\mu} D_{3}(y, a, b)=\left\{\begin{array}{l}
\vec{\Psi}^{(n)}(y, p)=\mathbf{y} D_{3}(y, a, b), \\
\left.\Psi^{(a}\right)(y, p)=y_{4} D_{3}(y, a, b)
\end{array}\right.
$$

Repeating as before all calculations we reduce the problem to find the coupling constant $\lambda=4 \alpha$ for given $\mu=\frac{\mu}{m}$ and $M=\frac{M}{m}$ to the variational 
task

$$
\lambda=\min _{a, b} \frac{\pi^{2} Z_{j}(\mu, a, b)}{J_{j}(\mu, M, a, b)}, \quad(j=n, a) .
$$

Here

$$
\begin{gathered}
Z_{n}(\mu, a, b)=\iint_{0}^{1} \frac{d t d u 18 u^{4}(1-u) t^{2}(1-t)^{2}}{\sqrt{b(b u+1-u)}\left(a^{2} u+\mu^{2}(1-u) t(1-t)\right)^{4}}, \\
Z_{a}(\mu, a, b)=\iint_{0}^{1} \frac{d t d u 6 u^{4}(1-u) t^{2}(1-t)^{2}}{\sqrt{b(b u+1-u)^{3}}\left(a^{2} u+\mu^{2}(1-u) t(1-t)\right)^{4}} \\
J_{j}(\mu, M, a, b)=\int_{0}^{\infty} d r \int_{0}^{\frac{\pi}{2}} d \theta \frac{r^{3} \cos ^{2}(\theta) V_{j}^{2}(r, \theta, a, b)}{\left(r^{2}+1-\frac{M^{2}}{4}\right)^{2}+M^{2} r^{2} \sin ^{2}(\theta)} \\
V_{n}(r, \theta, a, b)=\int_{0}^{1} \frac{d t t^{3}}{\sqrt{b t+1-t} \cdot \frac{4 r \cos (\theta)}{h(\mu, r, \theta, t, a, b)},} \\
V_{a}(r, \theta, a, b)=\int_{0}^{1} \frac{d t t^{3} \sqrt{b}}{\sqrt{(b t+1-t)^{3}} \cdot \frac{4 r \sin (\theta)}{h(\mu, r, \theta, t, a, b)},} \\
h(\mu, r, \theta, t, a, b)=\left[a^{2} t+\mu^{2}(1-t)+t(1-t) r^{2}\left(\cos ^{2}(\theta)+\frac{\sin ^{2}(\theta) b}{b t+1-t}\right)\right]^{3} .
\end{gathered}
$$

In Table 4 the results of calculations for "normal" states with spin $l=1$ and for "abnormal" states with spin $l=0$ are listed. The column $\lambda_{T j}$ contains the results obtained in [6] with factor $\frac{1}{2}$ to get the correct numbers. One can see that our numbers are quite close to them. It should be noted that in the strong coupling regime the "abnormal" states begin to play an essential role in the representation of the BS amplitude (13).

Acknowledgments. I would like to thank S.Dorkin for useful discussions and help in numerical calculations. This work was supported by the Russian Foundation for Basic Research under grant No. 01-02-17200-a. 
Table 4 . The first excited states in the case $\mu>0$.

\begin{tabular}{|c|c|c|c|c|c|c|c|c|}
\hline & & \multicolumn{4}{|c|}{ Vector particles $l=1$} & \multicolumn{3}{|c|}{ "Abnormal state" } \\
\hline$\frac{\mu}{m}$ & $\frac{M}{m}$ & $\lambda_{n}=4 \alpha$ & $a$ & $b$ & $\lambda_{T j}$ & $\lambda_{s}=4 \alpha$ & $a$ & $b$ \\
\hline 1.000 & 0.000 & 205.95 & 1.6 & 1.0 & & 205.95 & 1.6 & 1.0 \\
\hline 1.000 & 1.000 & 183.16 & 1.5 & 1.1 & & 195.35 & 1.5 & 1.1 \\
\hline 1.000 & 1.900 & 110.86 & 1.1 & 1.5 & & 166.26 & 1.3 & 1.3 \\
\hline 1.000 & 1.999 & 93.48 & 0.9 & 1.9 & & 161.66 & 1.3 & 1.5 \\
\hline 0.100 & 0.000 & 80.35 & 1.1 & 1.1 & 80.22 & 80.30 & 1.0 & 1.0 \\
\hline 0.100 & 1.000 & 63.77 & 0.9 & 1.2 & 63.77 & 63.74 & 0.9 & 1.1 \\
\hline 0.100 & 1.900 & 16.05 & 0.4 & 3.2 & 15.99 & 34.78 & 0.6 & 3.2 \\
\hline 0.100 & 1.999 & 4.87 & 0.2 & 6.2 & 4.77 & 27.44 & 0.4 & 4.4 \\
\hline 0.001 & 0.000 & 75.40 & 1.0 & 1.1 & & 75.40 & 1.0 & 1.0 \\
\hline 0.001 & 1.000 & 59.06 & 0.9 & 1.2 & & 64.24 & 0.9 & 1.2 \\
\hline 0.001 & 1.900 & 11.84 & 0.3 & 3.2 & & 25.75 & 0.4 & 4.1 \\
\hline 0.001 & 1.999 & 1.03 & 0.1 & 5.2 & & 10.11 & 0.05 & 15.0 \\
\hline
\end{tabular}

\section{$7 \quad$ Appendix. Angular polynomials $T_{\{\mu\}}^{(l)}(k)$}

The angular polynomials $T_{\{\mu\}}^{(l)}(k)=T_{\mu_{1}, \ldots, \mu_{l}}^{(l)}(k)$ are symmetric for $\mu_{i} \rightleftharpoons \mu_{j}$, and

$$
T_{\mu, \mu, \mu_{3}, \ldots, \mu_{l}}^{(l)}(k)=0
$$

The recurrence relation is

$$
T_{1 \ldots l}^{(l)}(k)=\frac{1}{l} P(1 \mid 2 \ldots l) k_{1} T_{2 \ldots l}^{(l-1)}(k)-\frac{k^{2}}{2 l(l-1)} P(12 \mid 3 \ldots l) \delta_{12} T_{3 \ldots l}^{(l-2)}(k)
$$


In particular

$$
\begin{aligned}
& T^{(0)}=1, \quad T_{1}^{(1)}(k)=k_{1}, \\
& T_{12}^{(2)}(k)=k_{1} k_{2}-\frac{1}{4} \delta_{12} k^{2}, \\
& T_{123}^{(3)}(k)=k_{1} k_{2} k_{3}-\frac{k^{2}}{6}\left(k_{1} \delta_{23}+k_{2} \delta_{31}+k_{3} \delta_{12}\right),
\end{aligned}
$$

The normalization condition is

$$
\sum_{\mu_{1} \ldots \mu_{l}} T_{\mu_{1}, \ldots, \mu_{l}}^{(l)}(k) T_{\mu_{1}, \ldots, \mu_{l}}^{(l)}(p)=\frac{|k|^{l}|p|^{l}}{2^{l}} C_{l}^{1}\left(\left(n_{k} n_{p}\right)\right),
$$

where $C_{l}^{1}(t)$ is the Gegenbauer polynomial.

These angular functions satisfy the formulas

$$
\square_{k} T_{\mu_{1}, \ldots, \mu_{l}}^{(l)}(k)=0, \quad\left(k \frac{\partial}{\partial k}\right) T_{\mu_{1}, \ldots, \mu_{l}}^{(l)}(k)=l T_{\mu_{1}, \ldots, \mu_{l}}^{(l)}(k) .
$$

\section{References}

[1] G.C.Wick, Phys.Rev., 96, 1124 (1954); R.E.Cutkosky, Phys.Rev., 96, 1135 (1954).

[2] C.Itzykson and J.-B.Zuber, Quantum Field Theory, McGraw-Hill Book Company, N.Y., 1980.

[3] J.R.Sapirstein and D.R.Yennie, Theory of Hydrogenic Bound States in Quantum Electrodynamics, Ed. T.Kinishita, Advanced Series on Directions in High Energy Physics, vol 7, World Scientific, 1999.

[4] W.Greiner and J.Reinhart, Quantum Electrodinamics, Springer-Verlag, Berlin, N.Y., 1992.

[5] N.Nakanishi, Suppl. of Pr.Theor.Phys., 43, 1 (1969).

[6] T.Nieuwenhuis, J.A.Tjon, Few Body Systems, 21, 167 (1996). 
[7] S.M.Dorkin, L.P.Kapteri and S.S.Semikh, Russ.Jad.Fiz, 60, 1784 (1997).

[8] S.H.Vosko, J.Math.Phys., 1, 505 (1960).

[9] C.Schwarz, Phys.Rev., 137B, 717 (1965).

[10] B.Simon, The $P(\phi)_{2}$ Euclidian (Quantum) Field Theory, Princeton Univ. Press, Princeton, 1974.

[11] N.N.Bogoliubov, D.V.Shirkov, Introduction to the Theory of Quantized Fields, Interscience Publishers, Ltd, N.Y., 1959.

[12] J.W.Darewych, Can.J.Phys., 76, 523 (1998). 